\title{
VERTICAL EMITTANCE IN THE KEK ACCELERATOR TEST FACILITY
}

\author{
T. Hirose, T. Okugi*, Y. Takayama, Tokyo Metro. Univ., Hachioji, Tokyo, Japan \\ H. Hayano, S. Kamada, K. Kubo, T. Naito, J. Urakawa, KEK, Tsukuba, Ibaraki Japan \\ S. Kashiwagi ${ }^{\dagger}$, The Graduate Univ. for Advanced Studies, Tsukuba, Ibaraki, Japan \\ M. Takano, Toho Univ., Funabashi, Chiba, Japan \\ M. Minty, J. Turner, M. Woodley, F. Zimmermann ${ }^{\ddagger}$, SLAC, Stanford, CA, USA
}

\begin{abstract}
We applied three methods in order to measure the vertical emittance of KEK-ATF. The first is a SR interferometer[1, 2], the second method is the emittance measurement by using wire scanners at the beam extraction line. The last method is emittance estimation by measuring an intensity dependence of a momentum spread, which is caused by the intra-beam scattering. The measured vertical emittance for KEK-ATF is $0.02-0.06 \mathrm{~nm}$, which is a few times larger than the design target of KEK-ATF.
\end{abstract}

\section{INTRODUCTION}

The horizontal emittance damped in the KEK-ATF damping ring was precisely measured by using the wire scanners at the beam extraction line. The measured horizontal emittance was $1.37 \pm 0.03 \mathrm{~nm}[3]$ at the bunch population of $3-5 \times 10^{9}$, which agreed well with the design value. On the other hand, the design target of the vertical emittance is $0.01 \mathrm{~nm}$, which corresponds to $1 \%$ of the horizontal emittance. Due to the vertical dispersion, a betatron coupling and other effects, it is hard to construct a damping ring with this extremely small vertical emittance. Furthermore, it is difficult to measure the vertical emittance itself due to the corresponding small vertical beam size both in the damping ring and at the extraction line. Due to these effects, we have currently not being able to measure the target value of the vertical emittance.

\section{EMITTANCE EXPECTATION}

\subsection{Vertical Dispersion}

Alignment errors of magnet devices generate an additional dispersion and a betatron coupling in a ring accelerator. The vertical dispersion, as a consequence, generates an additional vertical emittance component, which is generally larger than an ideal theoretical emittance. In order to produce a beam with extremely low vertical emittance, it is essential to reduce the vertical dispersion in the arc section of the accelerator. The relation between the dispersion and the emittance were simulated with the SAD code $[4,5]$. The emittance has linear dependence with the square of the

\footnotetext{
* Address will be changed to "KEK, Japan" from 1999 April.

$\dagger$ Address will be changed to "Waseda Univ., Japan” from 1999 April.

‡ Present address is "CERN, Switzerland".
}

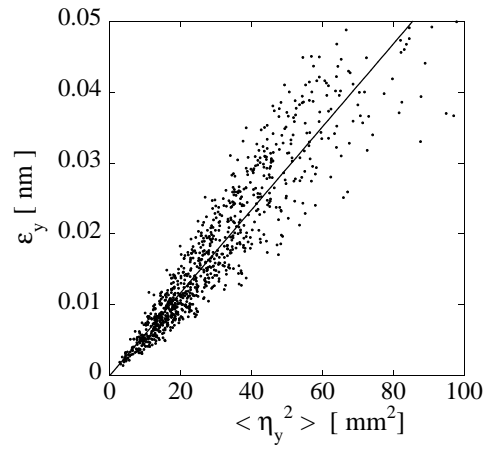

Figure 1: Simulation results for the vertical emittance of KEK-ATF damping ring as a function of the square of the vertical dispersion averaged over BPMs in the arc section.

vertical dispersion at the BPMs by

$$
\varepsilon_{y}[\mathrm{~nm}] \approx 0.0006\left\langle\eta_{y, \mathrm{BPM}}^{2}\left[\mathrm{~mm}^{2}\right]\right\rangle_{\mathrm{arc}},
$$

where the bracket means averaged over the arc section (see Figure 1). Furthermore, it was found from the simulation that the betatron coupling source does not create large vertical emittance enhancement for a KEK-ATF alignment accuracy[6]. Since the root mean square of the vertical dispersion was $4-9 \mathrm{~mm}$, the vertical emittance is expected to be 0.010-0.049 nm through 1998 November-1999 March beam operation period.

\subsection{Beam-gas Scattering}

There are two scattering effects for increasing the vertical emittance. One is beam-gas scattering and the other is intra-beam scattering. Both effects are prominent for a beam with low emittance and low energy, such as the KEKATF beam. The effect of beam-gas scattering on beam emittance was examined by deriving the beam distribution function for linear collider damping rings[7]. For reasonable vacuum pressures, the beam-gas scattering does not have a large effect on the core of the beam, but only a few particles are scattered to significant amplitudes. Figure 2 shows the effect of the beam-gas scattering on the vertical emittance for the KEK-ATF damping ring. Figure 2(a) shows the beam distribution function with the vertical emittance of $0.01 \mathrm{~nm}$ at several $\mathrm{CO}_{2}$ partial pressure. Figure 2(b) shows the corresponding vertical emittance as 

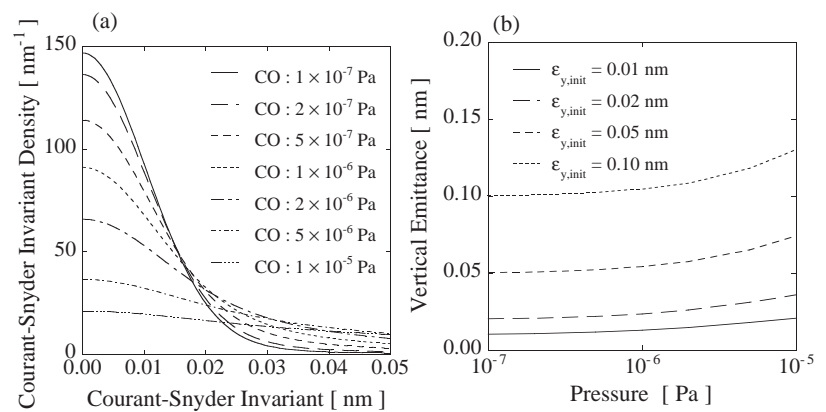

Figure 2: Effect of the beam-gas scattering. (a) Beam distribution function for the KEK-ATF beam with vertical emittance of $0.01 \mathrm{~nm}$. (b) Corresponding vertical emittance.

a function of $\mathrm{CO}_{2}$ partial pressure. Since the vacuum pressure of the KEK-ATF damping ring is less than $10^{-6} \mathrm{~Pa}$, the emittance enhancement by the beam-gas scattering is evaluated to be less than a few $10 \%$ of the emittance of the unscattered beam.

\subsection{Intra-beam Scattering}

Intra-beam scattering is an effect of a small scattering within the bunch, which are not sufficient to produce particle losses, but which nevertheless disturb the particle statistical distribution (noise source) [8, 9]. A spontaneous momentum deviation induces an energy oscillation around the nominal energy defined by the synchrotron radiation.

Since the bunch volume in the KEK-ATF damping ring is extremely small, it is expected that the effect of the intrabeam scattering has a strong influence on the momentum spread enhancement, as the consequence, on the vertical emittance enhancement. The effect of the intra-beam scattering on the momentum spread and the vertical emittance can be calculated by using a self-consistent equation for the KEK-ATF damping ring beam as shown in Figure 3. Since the intra-beam scattering is roughly proportional to the beam charge density, the enhancements are significant for higher beam intensities.

\section{EMITTANCE MEASUREMENT}

\section{$3.1 \quad$ SR Interferometer}

The SR interferometer is the beam size monitor by using the spatial coherence (visibility)[1,2]. This monitor has some advantages compared with other methods, because we do not have to pay attention to the field depth and the deflection limit of the SR light. Furthermore, since the validity for the application of the van Cittert-Zernike theorem to the beam in the KEK-ATF bending magnet field was confirmed[2], the SR interferometer is used as one of the most important emittance measurement tools in the KEKATF damping ring, especially as an online beam size monitor. The measured vertical beam size was $11-13 \mu \mathrm{m}$, which

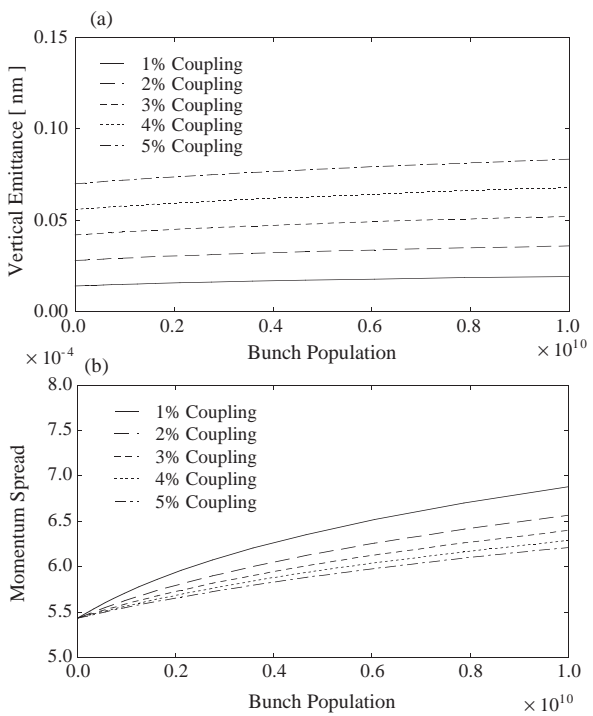

Figure 3: Calculation of the intra-beam scattering effect. (a) Effect on the vertical emittance. (b) Effect on the momentum spread.

corresponds to the vertical emittance of $\sim 0.05 \mathrm{~nm}$ through the 1998 November-1999 March beam operation.

\subsection{Wire Scanner}

In order to diagnose the beam properties, such as beam emittance, the damped beam is extracted to a beam extraction line in KEK-ATF. The beam optics of the KEK-ATF extraction line was designed to measure the vertical emittance by five wire scanners. Therefore, the beta function at each wire scanner was designed to be more than $5 \mathrm{~m}$ in order to make a large vertical beam size. Also, the phase advance between each wire scanner should be more than $10^{\circ}$ in order to measure with each individual monitor. A typical emittance result of the wire scanner is shown in Figure 4. In Figure 4(a), The circle represents the beam distribution in Floquet phase space. Five lines show the measured beam size boundaries converted to the same phase space. Figure 4(b) shows the measured beam size and expected beam
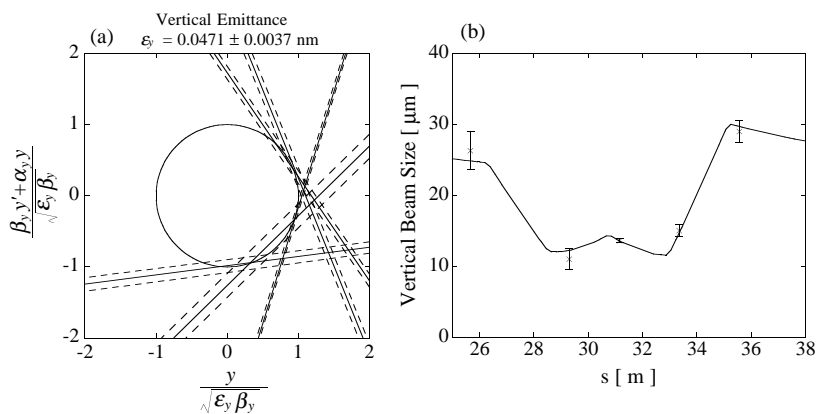

Figure 4: Typical emittance evaluation by wire scanner. (a) the measured beam size boundary at Floquet phase space, (b) measured beam size and expected beam size at beam diagnostic section. 


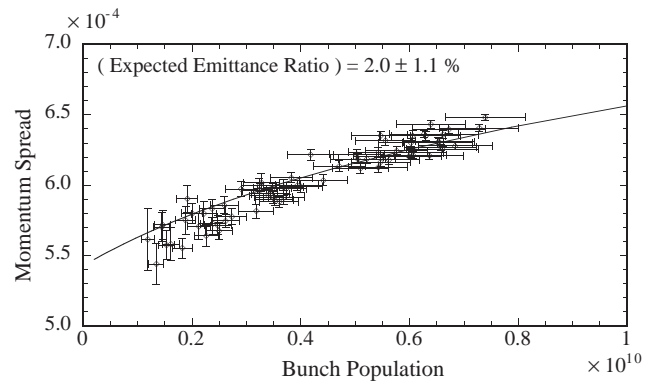

Figure 5: The intensity dependence of the momentum spread on the intra-beam scattering. Circles show the measured momentum spread and curve shows the calculated intensity dependence for the beam with the emittance ratio of $2.0 \%$.

size at the beam diagnostic section. The corresponding emittance value is $0.04-0.06 \mathrm{~nm}$ at the bunch population of $3-5 \times 10^{9}$ through the beam operation period.

\section{$3.3 \quad$ Intra-beam Scattering}

We applied one more vertical emittance estimation by using the intensity dependence of the momentum spread on the intra-beam scattering. This intensity dependence of the momentum spread was observed regularly during beam operation. The effect of the intra-beam scattering on a momentum spread enhancement was evaluated in Section 2.3. If we assume that the emittance ratio $\varepsilon_{y} / \varepsilon_{x}$ is constant, the measured beam intensity dependence of the momentum spread corresponds to an emittance ratio of $\varepsilon_{y} / \varepsilon_{x}=$ $2.0 \pm 1.1 \%$ (see Figure 5). Since the horizontal emittance is $1.37 \pm 0.03 \mathrm{~nm}$ at the bunch population of $3-5 \times 10^{9}[3]$, the emittance ratio measured above corresponds to a vertical emittance of $0.027 \pm 0.015 \mathrm{~nm}$ at this beam intensity.

\section{DISCUSSION}

The measured vertical emittance for each measurement was listed in Table 1. Each measured emittance was not far from the expectation value estimated from the observed vertical dispersion at the BPMs in the arc section of the KEK-ATF damping ring, however, it shows a slightly larger than the emittance expectation.

The results by SR interferometer and wire scanner were within the range of $0.04-0.06 \mathrm{~nm}$ at the bunch population of $3-5 \times 10^{9}$, and they were not reduced to less than 0.04 $\mathrm{nm}$ through the entire beam operation period, even when the apparent vertical dispersion was well suppressed in the arc section.

On the other hand, the vertical emittance evaluated by using the intensity dependence of the momentum spread is $0.027 \pm 0.015 \mathrm{~nm}$ at the same beam intensity, which was consistent with the expectation value of the vertical emittance, but smaller than the value found by the other methods. This difference has not been clarified yet. The difference might come from systematic errors of these mea-
Table 1: Summary of the vertical emittance in KEK-ATF.

\begin{tabular}{l|cc}
\hline \hline & Vertical Emittance & Charge \\
\hline Calculation & $0.010-0.049 \mathrm{~nm}$ & 0 \\
& $0.020-0.059 \mathrm{~nm}$ & $5 \times 10^{9}$ \\
\hline Measurement & & \\
SR Interferometer & $\sim 0.05 \mathrm{~nm}$ & $3-5 \times 10^{9}$ \\
Wire Scanner & $0.04-0.06 \mathrm{~nm}$ & $3-5 \times 10^{9}$ \\
Momentum Spread & $0.027 \pm 0.015 \mathrm{~nm}$ & $3-5 \times 10^{9}$ \\
\hline \hline
\end{tabular}

surement methods or come from the effect of the betatron coupling (beam tilt).

\section{SUMMARY}

The evaluated emittance is 2-6 times larger than the target vertical emittance of the KEK-ATF. One source of the large emittance was concluded as the vertical dispersion. We must reduce the vertical dispersion to less than $4 \mathrm{~mm}$ at the BPMs in the arc section of the damping ring in order to achieve the vertical emittance of $0.01 \mathrm{~nm}$. Furthermore, we need clarify the discrepancy between the emittance measurement and the emittance evaluation from the vertical dispersion measurement.

\section{ACKNOWLEDGEMENT}

The authors would like to thank Professors H. Sugawara, Y. Kimura, M. Kihara, K. Takata and S. Iwata for giving them the opportunity to do this study at the KEK-ATF and for their continuous support and encouragement. T. Okugi would also like to acknowledge that Tokyo Metropolitan University provides travel finding for this conference.

\section{REFERENCES}

[1] T. Mitsuhashi and T. Naito, Proc. of 6th European Particle Accelerator Conference, (1998) 1565.

[2] Y. Takayama et al., "Application Limit of SR Interferometer for Emittance Measurement", in this conference.

[3] T. Okugi et al., Phys. Rev. ST-AB, 2 (1999) 022801.

[4] K. Kubo et al., "OPTICS DIAGNOSTICS AND TUNING FOR LOW EMITTANCE BEAM IN KEK-ATF DAMPING RING', in this conference.

[5] hppt://www-acc-theory.kek.jp/SAD/sad.html.

[6] M. Takano et al., Proc. of 5th Int. Workshop on Accelerator Alignment, KEK Preprint 97-190 (1997).

[7] T. Raubenheimer, KEK Report $92-7$ (1992).

[8] J. Le Duff, CERN 95-06 (1995) 573.

[9] A. Piwinski, Proc. of 9th Int. Conf. on High Energy Accelerators, (1974) 405.

[10] T. Naito et al., "EMITTANCE MEASUREMENT AT KEKATF DAMPING RING", in this conference. 